\title{
Batch Hydrothermal liquefaction of end-of-life plastic and oil characterization
}

\author{
Benedetta Ciuffi ${ }^{1}$, Luca Rosi $^{1,2}$, Edoardo Miliotti ${ }^{2}$, Giulia Lotti $^{2}$, Andrea Maria Rizzo $^{2}$, David Chiaramonti ${ }^{2,3^{*}}$ \\ ${ }^{1}$ Chemistry Department "Ugo Schiff", University of Florence, Via della Lastruccia, 3-13 50019 Sesto Fiorentino, Florence, Italy \\ ${ }^{2}$ Renewable Energy COnsortium for R\&D (RE-CORD) Viale J. F. Kennedy, 182, 50038 Scarperia e San Piero, Italy \\ 3 "Galileo Ferraris" Energy Department, Polytechnic of Turin, Corso Duca degli Abruzzi 24, I-10129, Torino, Italy
}

\begin{abstract}
Finding a proper way to manage the enormous amount of waste plastic that is globally produced, is one of the main environmental challenges of our times. Among the different types of chemical recycling, Hydrothermal Liquefaction (HTL) appears as a potential method for the treatment of plastic waste mixes, for sustainable production of biocrude or chemicals with high added value. In this work hydrothermal liquefaction reactions were carried out on a polymeric residue, obtained from an industrial plastic waste collection and recycling process. The residue has a heterogeneous composition consisting not only of polymers but also paper and metals. Two batch experiments were performed in a stainless-steel Parr autoclave at $340{ }^{\circ} \mathrm{C}$, investigating a residence times of 5 hours and the use of an alkaline catalyst $(\mathrm{NaOH})$. The oils obtained from the reactions, as well as the aqueous phases, have been analysed by different analytical techniques such as: FT-IR spectroscopy, GC-MS, GC-FID, IC. The operating conditions used in this work, allowed the degradation of cellulose and polymers with reactive sites for hydrolysis such as PET, nylon and PVAc, while polyolefins (PE, PP) were not attacked. The use of a basic catalyst favoured a greater hydrolysis rate.
\end{abstract}

\section{Introduction}

The multifunctionality and the relatively low cost of plastic, make it an omnipresent material in everyday life and its global production is continuously increasing year after year. In 2018 the production of plastic increased by $3 \%$ compared to the previous year [1], reaching 359 million tons. Packaging by far represents the largest end-use market [2]. Most conventional plastics such as polyethylene (PE), polypropylene (PP), polystyrene (PS), polyvinyl chloride (PVC) and polyethylene terephthalate (PET), are non-biodegradable and their increasing accumulation in the environment has been a threat to the planet [3-5].

To solve the problem related to plastics pollution, with the directive UE 2019/904 [6], Europe has imposed that from 2021 some disposable packaging and products should be prohibited. The directive also promotes the circular economy principle, which aims to keep resources in use for as long as possible, to extract the maximum value from them whilst in use, and to recover and regenerate products and materials at the end of their service life [7]. In this context, plastic waste can be recycled for the remanufacturing of new plastics or conversion into other valuable products. Among the different types of chemical recycling, Hydrothermal Liquefaction (HTL) appears as a very promising method for the sustainable production of biocrude [8]. This technique has high potential as an environmentally friendly green process due to the superior properties as chemical reaction media [9]. In this process, water is commonly used as solvent. Particular chemicalphysical properties are observed near the critical point, defined by a certain value of temperature and pressure. For water, the critical point is defined by a temperature of $374.1{ }^{\circ} \mathrm{C}$ and a pressure of $22.1 \mathrm{MPa}$ [10]. When water is above boiling point but below the critical point was defined as subcritical water. Water under supercritical or subcritical conditions is an excellent reaction medium for depolymerisation and decomposition of plastics. Polymers decomposition occurs selectively and quickly. In particular, water in sub-super-critical conditions has excellent thermal and chemical properties and it is an effective medium for processing technically difficult waste, such as mixed types of plastics, and plastic contaminated with organics, which are otherwise incinerated $[11,12]$. The flexibility of the hydrothermal process allows the

\footnotetext{
${ }^{*}$ Corresponding author: david.chiaramonti@polito.it
} 
processing of different plastics regardless of color, sizes, purity, physical properties, within the same process [11]. Starting from our knowledge in the field of HTL on biomass, in this work we used the hydrothermal liquefaction reaction to study a real processing residue of plastic waste, coming from a recycling plant in northern Italy. This residue has a heterogeneous composition consisting of a large variety of polymers, paper and metals. It was used without further purification. Two reactions were carried out working under subcritical conditions $\left(340^{\circ} \mathrm{C}, 5 \mathrm{~h}\right)$, as suggested by recent literature [8]. Since a significant part of the feedstock was PET, a test was conducted with $2 \mathrm{wt} \%$ of $\mathrm{NaOH}$, to facilitate the breakdown of ester bonds, as occurs in basic hydrolysis processes [13]. The yields, as well as the composition of the oils and aqueous phases obtained in the two tests were compared. To the best of the Authors knowledge this is the first study about a real plastic residue containing not only polyolefins but a mix of different waste polymers, paper and metals.

\section{Materials and method}

\subsection{Feedstock}

The feedstock used in this work, was a polymeric compound, obtained from an industrial plastic waste collection and recycling process. In particular, it came from the separate collection of plastic waste.

\subsection{Experimental equipment and procedure}

The hydrothermal liquefaction experiments were performed in a $160 \mathrm{~mL}$ stainless steel Parr autoclave. The autoclave is equipped with a stirrer, a pressure sensor and a J-type thermocouple. The heating system consists of a $1 \mathrm{~kW}$ electric band heater regulated by a PID controller. In the first test the reactor was filled with $5 \mathrm{~g}$ of feedstock and $50 \mathrm{~g}$ of ultrapure water $(0.05 \mu \mathrm{S}$ $\left.\mathrm{cm}^{-1}\right)$. The second test was performed in a basic environment, adding 2 wt.\% of $\mathrm{NaOH}$ solution; a leakage test with Argon at 80 bar was performed and then three purging cycle with Nitrogen (5 bar) were carried out in order to ensure an inert atmosphere. Reaction temperature was set to $340^{\circ} \mathrm{C}$ and the average heating rate was $7^{\circ} \mathrm{C} \mathrm{min}{ }^{-1}$. After $5 \mathrm{~h}$ of residence time at reaction temperature and under stirring at $245 \mathrm{rpm}$, the autoclave was rapidly cooled by immersion in a cold-water bath.

Reaction products were collected by means of the procedure reported in figure 1. The reactor and its content were washed with dichloromethane (DCM) and then filtered under vacuum over a glass microfiber filter (1 $\mu \mathrm{m})$. The DCM-insolubles were oven-dried at $105^{\circ} \mathrm{C}$ until constant weight, while the aqueous phase and the DCM-solubles were gravimetrically separated. The dichloromethane phase was then subjected to evaporation under reduced pressure and the so-called oil was collected.

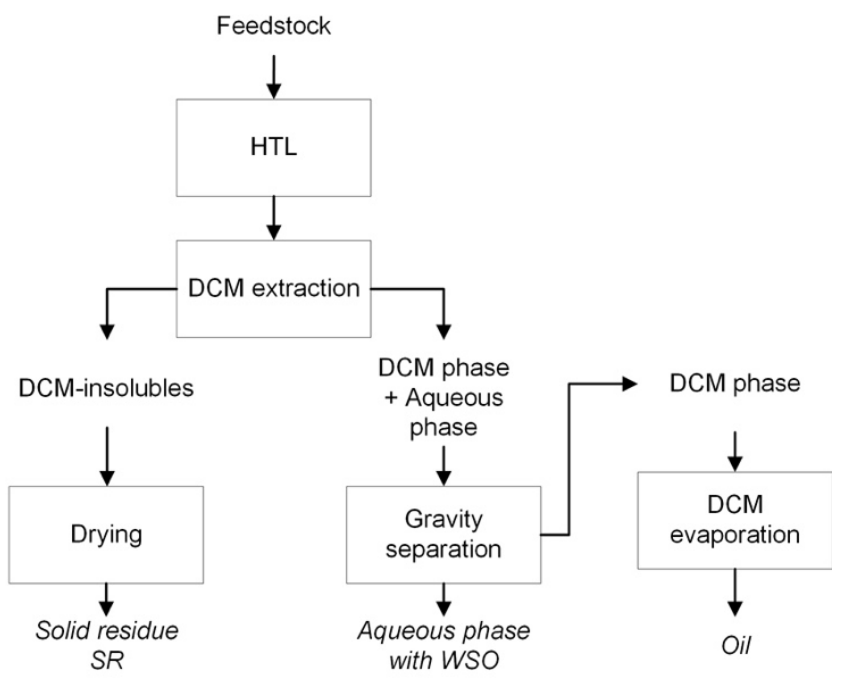

Fig. 1. Collection procedure of HTL products (DCM stands for dichloromethane and WSO for Water-Soluble Organics).

\subsection{Determination of the yields}

For each test, yields of solid residue, gas, oil products and Water-Soluble Organics + unrecovered (WSO+ Unre) were determined by equations 1-4:

$$
\begin{aligned}
& \text { Solid residue yield }(\%)=\frac{W_{\text {residue }}}{W_{\text {Feedstock }}} \times 100 \\
& \text { Oil product yield }(\%)=\frac{W_{\text {oil }}}{W_{\text {Feedstock }}} \times 100
\end{aligned}
$$

Gas yield $(\%)=$ Final reactor weight Initial reactor weight

$$
\begin{aligned}
& \text { WSO }+ \text { Unre }(\%)=100-(\text { solid yeld }+ \text { oil yield } \\
& + \text { gas yield })
\end{aligned}
$$

\subsection{Analytical methods and chemicals}

Thermogravimetric analysis was performed in a Leco TGA 701 instrument equipped with ceramic crucibles. Temperature was raised from ambient to $650^{\circ} \mathrm{C}$ under a

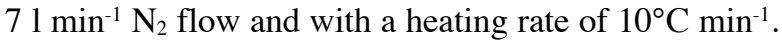
FTIR spectra of feedstocks and reaction products were obtained using a Shimadzu IRTracer-100 spectrometer, equipped with QATR ${ }^{\mathrm{TM}} 10$ Single-Reflection ATR with a Diamond Crystal, operating with a maximum resolution of $0.25 \mathrm{~cm}^{-1}$ and a spectral range in mid-IR region (4000-600 $\left.\mathrm{cm}^{-1}\right)$.

Qualitative and quantitative analysis of the organic compounds in the oil and aqueous samples were performed by GC-MS and GC-FID, respectively, both equipped with a Zebron ZB-5HT INFERNO (Phenomenex) column (length $30 \mathrm{~m}$, internal diameter $0.250 \mathrm{~mm}$, film diameter $0.25 \mu \mathrm{m}$ ).

About $0.1 \mathrm{~g}$ of oil was dissolved in $10 \mathrm{ml}$ of dichloromethane and then $2 \mu \mathrm{l}$ of the solution was injected in a GC-MS apparatus (GC 2010 with a GCMSQP2010 mass spectrometer, Shimadzu). The analysis was performed with a helium column flow of $2.02 \mathrm{ml}$ 


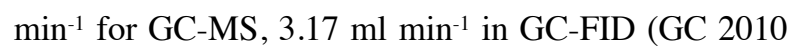
plus Shimadzu) with an initial temperature of $40{ }^{\circ} \mathrm{C}$ (holding time $10 \mathrm{~min}$ ), increased to $200^{\circ} \mathrm{C}$ (heating rate $8^{\circ} \mathrm{C} \mathrm{min}^{-1}$, hold time $10 \mathrm{~min}$ ) and then to $280^{\circ} \mathrm{C}$ (heating

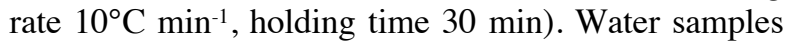
were injected after a 1:1 dilution in iso-propanol.

The GC-MS apparatus was used to determine the qualitative composition of the sample by comparing the acquired spectra against those of NIST 17 library; the GC-FID apparatus was used instead for the quantitative determination of identified compounds after a previous 4 points calibration with pure molecular standards and using o-terphenyl as internal standard. The same temperature profile of the GC-MS was adopted.

The determination of chloride content $\left(\mathrm{Cl}^{-}\right)$on aqueous samples was performed in an Ion Chromatographic system 883 Basic IC Plus (Metrohm), equipped with a conductivity detector, according to EN ISO 10304 after a $0.2 \mu \mathrm{m}$ syringe filtration and a 4-point calibration with pure standard.

\section{Results and discussion}

\subsection{Yields}

The reactions yields are shown in table 1 .

Table 1. Reactions yields

\begin{tabular}{|c|c|c|}
\hline & Test 1 & Test 2 (NaOH) \\
\hline Oil & $7.7 \%$ & $7.4 \%$ \\
\hline Solid & $75.1 \%$ & $65.5 \%$ \\
\hline Gas & $11.0 \%$ & $11.0 \%$ \\
\hline WSO + Unre & $6.2 \%$ & $16.0 \%$ \\
\hline
\end{tabular}

A comparison between the yields of the two tests, shows that, the addition of $\mathrm{NaOH}$, leads to a decrease in the percentage of solid residue and an increase in the percentage of WSO.

\subsection{Feedstock characterization:}

The feedstock used in this work, was characterized by thermogravimetric analysis and FT-IR spectroscopy.

\subsubsection{Thermogravimetric analysis (TGA)}

The derivative of the mass loss of the feedstock (DTGA curve) is reported in figure 2 . The thermogram shows three-steps of mass loss: the first at about $125^{\circ} \mathrm{C}$, corresponding to a mass loss of around $1.3 \mathrm{wt} . \%$, which can be attributed to the loss of moisture in the sample; the second at about $300^{\circ} \mathrm{C}$, corresponding to a further $6.5 \mathrm{wt} . \%$ of mass loss; and a last step of degradation at about $525^{\circ} \mathrm{C}$, corresponding to a mass loss of around 69 wt. $\%$. At the end of the scan $\left(650^{\circ} \mathrm{C}\right)$, about $23.2 \%$ of non-degraded residual mass remains, which can be associated with the presence of charred plastics, metals or inorganic salts.

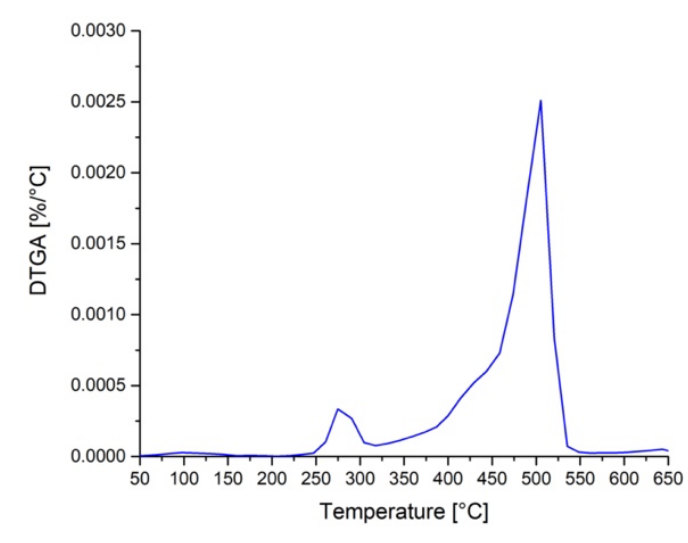

Fig. 2. Derivative of feedstock mass loss

\subsection{FT-IR spettroscopy}

The feedstock appears to be a highly heterogeneous material. A first simple separation of the components was carried out using the principle of flotation. This method is based on the different specific weight (SW) of the various materials constituting the feedstock: those with SW higher than that of water, sink ( $S W>1$, styrenebase polymers or PVC or PET) while those with SW lower than that of water, float $(\mathrm{SW}<1$, polyolefin-base polymers). Our sample was thus separated into two fractions: the first afloat containing dark grey plastic and paper; the second sunken and made up of fragments of colored plastics scales and metals. The IR spectra acquired on the first fraction are reported in figure 3 . They show characteristic absorption of polyethylene (2916 cm $\mathrm{cm}^{-1}, 2848 \mathrm{~cm}^{-1}, 1471 \mathrm{~cm}^{-1}, 721 \mathrm{~cm}^{-1}$ ) [14]. However, unexpected absorptions for polyolefins appear, such as a signal of medium intensity at $1718 \mathrm{~cm}^{-}$ 1 related to $\mathrm{C}=\mathrm{O}$ stretching. Signals related to $\mathrm{C}-\mathrm{O}$ stretching $\left(1251 \mathrm{~cm}^{-1}, 1101 \mathrm{~cm}^{-1}\right)$ and $\mathrm{C}=\mathrm{C}$ bending (1018 $\mathrm{cm}^{-1}$ and $875 \mathrm{~cm}^{-1}$ ) also appear. As we could expect from the nature of the sample, the first fraction is not made up of pure polyolefin, but is contaminated by aromatic polymers containing oxygen, such as PET. The IR spectra acquired on the first fraction also confirm the presence of cellulose from the paper.

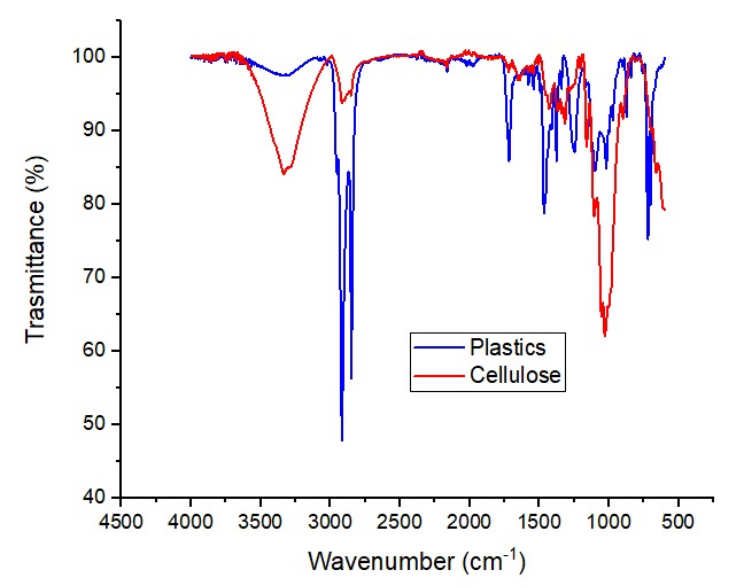

Fig. 3. IR spectra of the floating fraction of the sample 
The sunken fraction is made up of plastics fragments of different colours (green, blue, white, orange, black) and hardness. An IR spectrum was acquired for each of them to obtain information on their composition. Most of the components shows the typical absorptions of PET.

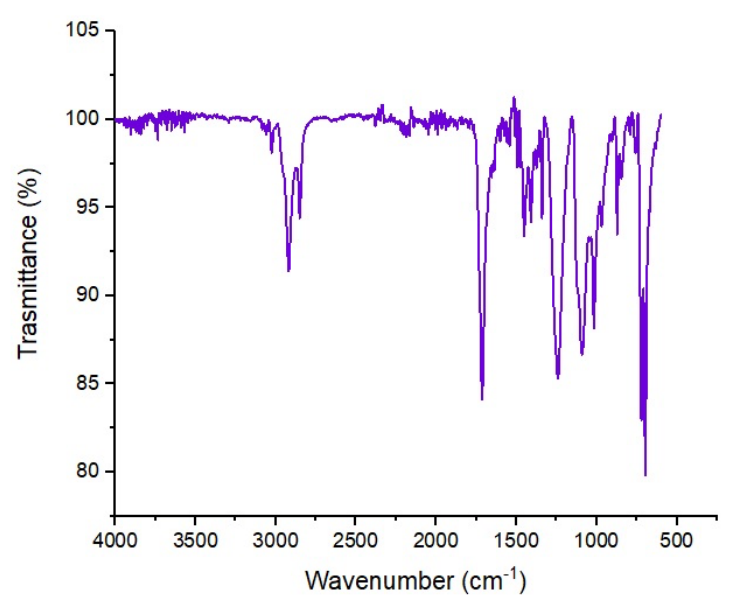

Fig. 4. IR spectrum of PET-DLA fragments present in the feedstock.

In particular, a comparison between the IR spectra obtained experimentally on PET fragments (figure 4), with IR spectra from literature, suggests that the scraps are made up of PET-DLA copolymer [15]. Dimer fatty acids, such as dilinoleic acid (DLA) or their diols, are widely used as components of commercially available adhesives, coating materials or polyurethanes. They are also successfully used for modification of polyamides or polyesters, for producing thermoplastic elastomers (TPE) [16].

In particular, a copolymer composed of PET hard segments and DLA soft segments (see figure 5 for the structural formula) shows excellent mechanical properties and it is susceptible to hydrolytic degradation [16].

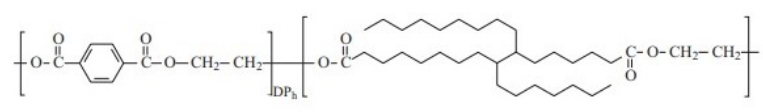

Fig. 5. Structure of a PET-DLA copolymer

An absorption at about $698 \mathrm{~cm}^{-1}$ relatives to the $\mathrm{C}-\mathrm{Cl}$ bond appears in the spectrum of figure 4 . It is probably due to the presence of PVC. The IR spectra on the remaining fragments (not shown in the paper), depict characteristic absorption of other polymers such as polystyrene, bakelite, polyvinyl acetate, nylon.

\subsection{Oil characterization}

Oil products were analysed qualitatively by GC-MS and FT-IR and quantitatively by GC-FID.

The IR spectrum of the oil from test 1 (blue curve) was reported in figure 6 , together with the IR spectrum of the oil obtained from test 2 (red curve). The absorptions in the range between 2960 and $2855 \mathrm{~cm}^{-1}$ are related to the $\mathrm{C}-\mathrm{H}$ stretching of alkanes. These signals are superimposed on a broadband in the region between 2500-3300 $\mathrm{cm}^{-1}$, due to the O-H stretching of carboxylic acids. The spectrum underlines the presence of oxygencontaining compounds $\left(1688 \mathrm{~cm}^{-1} \mathrm{C}=\mathrm{O}\right.$ stretching; 1488 $\mathrm{cm}^{-1} \mathrm{O}-\mathrm{H}$ bending, $1252 \mathrm{~cm}^{-1} \mathrm{C}-\mathrm{O}$ stretching) and aromatic rings $\left(706 \mathrm{~cm}^{-1} \mathrm{C}-\mathrm{H}\right.$ bending of monosubstituted benzene derivatives).

GC-MS analysis of the oil from test 1 (figure 7), reveals the presence of a high amount of benzoic acid (18\% $\mathrm{w} / \mathrm{w}$ ), direct product of terephthalic acid (TA) decarboxylation [8]. TA comes from the hydrolysis of PET. As expected from IR spectrum, the analysis highlights the presence of a $2.84 \% \mathrm{w} / \mathrm{w}$ of oxygenated compounds. The oil contains monosubstituted benzene derivatives $(1.09 \% \mathrm{w} / \mathrm{w})$ and compounds containing more than one aromatic ring $(2.33 \% \mathrm{w} / \mathrm{w})$. These products are related to the degradation of polystyrene in the feedstock. Phenol $(0.41 \% \mathrm{w} / \mathrm{w})$ is assumed to derive from Bakelite degradation. Through the combined use of GC-MS and GC-FID, 25.6\% w/w of the compounds in the oil were characterized and quantified.

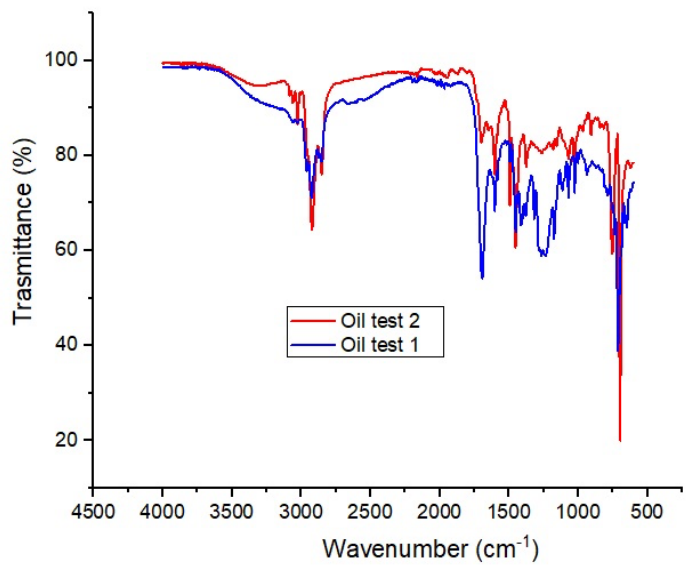

Fig. 6. Comparison between the IR spectra of the oils obtained in the two tests

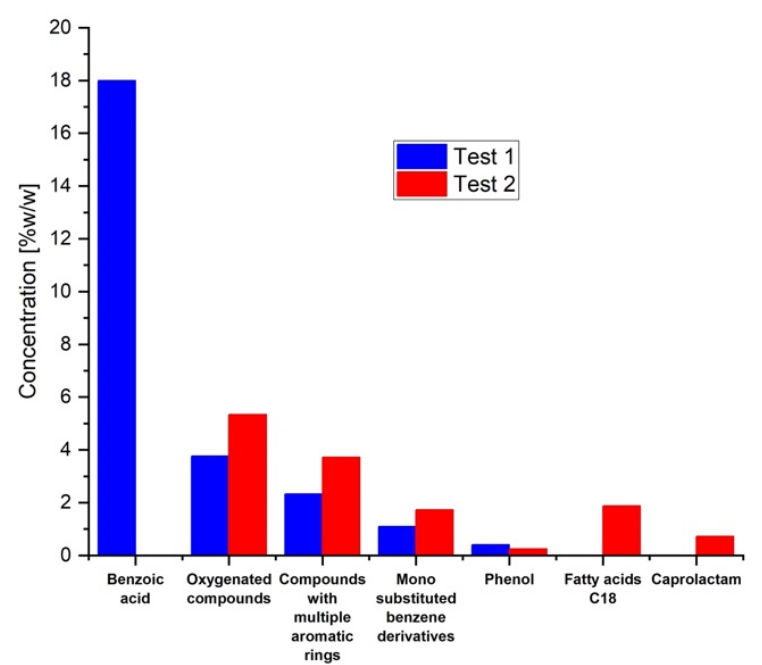

Fig. 7. Mass composition of oil from test 1 (blue) and oil from test 2 (red)

The IR spectrum of the oil obtained from test 2 appears very similar to the previous one. The broadband 
associated with the $\mathrm{O}-\mathrm{H}$ stretching of benzoic acid disappeared and appears a narrower band at $3300 \mathrm{~cm}^{-1}$ relatives to $\mathrm{O}-\mathrm{H}$ stretching of alcohols. The absorption related to $\mathrm{C}=\mathrm{O}$ appears less intense than in the previous case, while the absorption linked to $\mathrm{C}-\mathrm{O}$ stretching completely disappear. Absorptions related to $\mathrm{C}=\mathrm{C}$ and $\mathrm{C}-\mathrm{H}$ bendings of aromatic compounds $\left(1490 \mathrm{~cm}^{-1}, 1452\right.$ $\mathrm{cm}^{-1}, 753 \mathrm{~cm}^{-1}$ ) are observed in the spectrum.

The GC-MS analysis (figure 7), reveals how the use of an alkaline catalyst favors the breaking of ester bonds in PET-DLA, allowing a greater fragmentation of the copolymer, confirmed by the presence of $\mathrm{C} 18$ fatty acids $(1.88 \% \mathrm{w} / \mathrm{w})$. The presence of benzoic acid is not revealed, probably due to the formation of high molecular weight benzoates. Sodium hydroxide also causes an increase in oxygenated compounds $(5,34 \%$ $\mathrm{w} / \mathrm{w})$ and compounds containing in their structure more than one benzene ring $(3,72 \% \mathrm{w} / \mathrm{w})$. As suggested by literature [8], basic catalysts promote the decomposition of nylon, giving rise to caprolactam $(0.72 \% \mathrm{w} / \mathrm{w})$. Through the combined use of GC-MS and GC-FID, $13.6 \% \mathrm{w} / \mathrm{w}$ of the compounds in the oil were characterized and quantified.

For both oils, the GC-MS analysis does not show the presence of C6-C11 paraffins and C6-C13 olefins, typical products of the degradation of polyolefins [17]. This result is in agreement with the literature [8], since supercritical conditions are required for polyolefins degradation [17].

\subsection{HTL water phase characterization}

A $\mathrm{pH}$ measurement was conducted on the aqueous phases after extraction with DCM. The aqueous phase coming from test 1 has a $\mathrm{pH}$ of 4.9 , while the one coming from test 2 has a $\mathrm{pH}$ of 8.4.

The observed $\mathrm{pH}$ values are related to the formation of organic acids, which were revealed by the GC-MS analysis. In the aqueous phase coming from test 1 , acetic acid $\left(847 \mu \mathrm{g} \mathrm{ml}^{-1}\right)$, benzoic acid $\left(396 \mu \mathrm{g} \mathrm{ml}^{-1}\right)$ and propionic acid $\left(142 \mu \mathrm{g} \mathrm{ml}^{-1}\right)$ were present. Acetic acid is a characteristic product of the hydrolysis of PVAc. In the aqueous phase coming from test 2, the GC-MS analysis reveals the presence of propionic acid $(177 \mu \mathrm{g}$ $\left.\mathrm{ml}^{-1}\right)$ and dihydroxyacetone $\left(1431 \mu \mathrm{g} \mathrm{ml}^{-1}\right)$. This latter compound, deriving from the degradation of cellulose [18], gives the solution a dark orange color.

Ion chromatography reveals the presence of a high quantity of chlorides in both aqueous phase (test 1: 2549 ppm; test 2: $2517 \mathrm{ppm}$ ), due to the presence of PVC in the feedstock.

\section{Conclusion}

During this work hydrothermal liquefaction reactions were carried out on real polymeric residue, obtained from an industrial plastic waste collection and recycling process. The operating conditions used in this work (340 ${ }^{\circ} \mathrm{C}, 5 \mathrm{~h}$ ), allowed the degradation of cellulose and polymers with reactive sites for hydrolysis such as PET, nylon and PVAc, while polyolefins (PE, PP) were not attacked. The use of a basic catalyst favored hydrolysis, leading to a decrease in the solid residue and an increase in the WSO, as expected from literature [8]. In the future it will be necessary to conduct further tests, varying the reaction time and the percentage of catalyst, to identify the best process conditions.

\section{Acknowledgements}

Authors wish to acknowledge Eng. Arturo di Fraia for his valuable help in performing the HTL experiments.

\section{References}

[1] PlasticsEurope, "Plastics-the Facts 2018". https://www.plasticseurope.org/application/files/63 15/4510/9658/Plastics_the_facts_2018_AF_web.pd f.

[2] PlasticsEurope, "Plastics - the Facts 2019". https://www.plasticseurope.org/application/files/97 15/7129/9584/FINAL_web_version_Plastics_the_f acts2019_14102019.pdf.

[3] Y. Tokiwa, B. P. Calabia, C. U. Ugwu, S.Aiba, Int. J. Mol. Sci., 10, no. 9, 3722-3742 (2009)

[4] G. Reimonn, T. Lu, N. Gandhi, W. T. Chen, J Renew Mater, 7, no. 12., 1251-1268 (2019)

[5] G. Ašmonaite and B. C. Almroth, no. February, 171 (2019)

[6] Parlamento Europeo e del Consiglio, "Direttiva (UE) 2019/904," https://eurlex.europa.eu/legalcontent/IT/TXT/PDF/?uri=CELEX:32019L0904\&f rom $=\mathrm{EN}$.

[7] STAP, "Plastics and the circular economy" https://stapgef.org/sites/default/files/publications/P LASTICS for posting.pdf.

[8] J. S. dos Passos, M. Glasius, P. Biller, Process Saf. Environ. Prot. (2020)

[9] M. Goto, J. Japan Pet. Inst., 59, no. 6, 254-258 (2016).

[10] M. Möller, P. Nilges, F. Harnisch, U. Schröder, ChemSusChem, 4, no. 5., 566-579 (2011).

[11] T. Helmer Pedersen and F. Conti, Waste Manag., 68, pp. 24-31 (2017).

[12] M. R. Gent, M. Menendez, J. Toraño, I. Diego, Waste Manag Res, 27, no. 2., 175-187 (2009)

[13] A. M. Al-Sabagh, F. Z. Yehia, G. Eshaq, A. M. Rabie, A. E. ElMetwally, Egypt. J. Pet., 25, no. 1, 53-64 (2016).

[14] J.V Gulmine, P.R Janissek, H.M Heise, L Akcelrud, Polym. Test., 21 (2002)

[15] A. Niemczyk, P. Kaczorowski, M. El Fray, Prog. Chem. Appl. Chitin its Deriv., 20, 236-245 (2015)

[16] A. Piegat, M. Eł Fray, Polimery/Polymers, 52, no. 11-12, 885-888 (2007)

[17] W. T. Chen, K. Jin, N. H. Linda Wang, ACS Sustain. Chem. Eng., 7, no. 4, 3749-3758 (2019)

[18] S. Yin, Z. Tan, Appl. Energy, 92, 234-239 (2012) 